\title{
Representaciones sociales de la influenza humana de adolescentes de la ciudad de Guadalajara, México
}

\author{
Social Representations that Adolescents from Guadalajara, Mexico \\ Have About Human Influenza
}

\author{
Teresa Margarita Torres-López ${ }^{1}$ \\ Yocelin Celeste Núñez-Sandoval ${ }^{2}$ \\ Antonio de Jesús De la Cruz-Villarreal ${ }^{3}$ \\ Universidad de Guadalajara, México
}

Resumen. El objetivo del estudio es analizar las representaciones sociales (RS) de la influenza humana que poseen adolescentes de Guadalajara, México, durante la epidemia del 2009 y ante la presencia de un brote a inicios del 2016. Se aplicó la técnica asociativa de listados libres a 112 adolescentes, para conocer la estructura de las RS a través de un análisis prototípico y el contenido con un análisis procesual. Los hallazgos muestran que las RS de la influenza en el 2009 se encontraban en proceso de construcción; son contradictorias, al calificarla de peligrosa a la vez que se le considera una mentira o exageración. Por otro lado, en 2016, las RS parecen haberse resignificado en torno a los síntomas, atención y posibles consecuencias.

Palabras clave. Representaciones sociales, influenza, adolescentes, Psicología de la Salud.

Abstract. The aim was to analyze social representations (SR) that adolescents from Guadalajara, Mexico have about human influenza, during the epidemic of 2009 and in early 2016 in the presence of an influenza outbreak. Free listings associative technique was applied to 112 adolescents, in the interest of knowing the RS structure by a prototypical analysis and the thematic content by procesual analysis. The findings show that influenza SR in 2009 was on construction process. It is contradictory because they underline its danger, and at the same time they point the influenza as a lie or exaggeration. In 2016 it seems to have redefined around symptoms, care and possible consequences.

Keywords. Social representations, influenza, adolescents, health psychology.

\footnotetext{
${ }^{1}$ Teresa Margarita Torres López. Universidad de Guadalajara, México. Dirección Postal: Calle Sierra Mojada 975, puerta 1. Colonia Independencia, Guadalajara, Jalisco, México, C.P. 44340.E-mail: tere.torres.cucs@gmail.com

${ }^{2}$ Yocelin Celeste Núñez Sandoval. Universidad de Guadalajara, México. E-mail: yocelin_233@hotmail.com

${ }^{3}$ Antonio de Jesús Cruz Villarreal Universidad de Guadalajara, México. E-mail: ajcv90@gmail.com
}

\section{@ $\odot \Theta \Theta$}




\section{Introducción}

A partir de la alerta mundial por la difusión de un nuevo virus causante de influenza (identificado por las siglas A (H1N1)), durante el mes de abril de 2009, se han realizado numerosos estudios epidemiológicos y clínicos al respecto. Se identificó a México como el país de origen de esta, lo cual se reafirmó con el reporte de casos confirmados (Organización Mundial de la Salud, OMS, 2009). Para finales del año, se tenía un total de 68 611 casos en dicho país; mientras que, para finales del año 2010, se reportaron 2453 casos (Secretaría de Salud de México, 2011). Durante el año 2015, la mayoría de los Estados Miembros del Caribe registraron baja actividad de influenza y de otros virus respiratorios. En Costa Rica, la actividad de influenza se incrementó en 2015. $\mathrm{El}$ virus predominante fue influenza A (H1N1), seguido de influenza A (H3N2) (Organización Panamericana de la Salud, OPS-OMS, 2016).

Ante este escenario, la OMS destaca la importancia de incluir a las disciplinas de las ciencias sociales, para ampliar la comprensión de por qué las personas tienen ciertas prácticas de prevención para algunas enfermedades y no para usarlas en otras, a pesar de la información que se difunde con respecto a los beneficios o peligros de su conducta (OMS, 2014). Dicho entendimiento permitiría promover la comunicación oportuna para conseguir que la comunidad participe y para evitar la confusión y la desinformación (OPS, 2011).

La Psicología de la Salud se propone como una disciplina que puede dar respuesta a estas demandas, ya que entre sus objetivos se encuentran los de promoción y mantenimiento de la salud; y la prevención y el tratamiento de la enfermedad a través de la modificación de hábitos. Los estudios desarrollados en esta disciplina permiten la construcción de modelos explicativos desde una perspectiva interdisciplinaria, considerando el contexto cultural y sanitario en el cual se presenta la enfermedad. Por ello, su papel en la planificación de acciones preventivas de salud es muy importante, al destacar que estas deben tomar en cuenta los valores sociales y los significados del grupo social afectado (Oblitas, 2006).
El caso específico de la influenza debe ser abordado desde diversas ópticas, resaltando la enseñanza que dejó, para contar con bases más firmes para el desarrollo de futuras intervenciones. El presente estudio aporta la visión de adolescentes mexicanos, futura población adulta, cuyas representaciones sociales (RS) podrían orientar sus conductas de protección, prevención y atención ante la presencia de enfermedades emergentes.

Se trata de una comparación de dos grupos de adolescentes, con información levantada en el 2009, durante la pandemia de la influenza humana, con otra recabada a inicios del 2016, cuando se presentó un brote de influenza estacional que incluyó diferentes cepas, entre otras la A (H1N1), A (H3N2), influenza A y B. En México, en el año 2016, el mayor número de casos se registró en los meses de febrero a marzo (semanas del 14 de febrero al 12 de marzo) con 5617 casos confirmados y 346 defunciones (Secretaría de Salud de México, 2016).

Una perspectiva teórica y metodológica que permite conocer más de cerca el concepto de influenza que posee la población adolescente, dentro de un marco cultural, son las RS. Algunos elementos que han sido considerados parte de estas son las creencias, los saberes de sentido común y el papel del ser humano como productor de conocimientos (Jodelet, 1993). El concepto de RS se define como una forma de conocimiento específico, de saber de sentido común, cuyos contenidos manifiestan la operación de procesos generativos y funcionales socialmente caracterizados; en sentido más amplio, designan una forma de pensamiento social y constituyen modalidades de pensamiento práctico orientados hacia la comunicación, la comprensión y el dominio del entorno social, material e ideal (Jodelet, 1993).

Existen dos formas de acercamiento al estudio de las RS; el enfoque estructural y el procesual. En el primero, los contenidos de la RS son considerados como estructuras organizadas. Se caracteriza por "identificar su estructura o su núcleo y por desarrollar explicaciones acerca de las funciones de esta estructura"(Abric, 2001). Hace énfasis en el estudio del núcleo central o sistema que da significado a la representación social y 
del periférico, comprende los elementos de la RS que están influenciados por el contexto social inmediato en el que los sujetos viven (Abric, 2001). El enfoque procesual identifica a las RS como procesos discursivos, caracteriza al ser humano como productor de sentidos y focaliza su análisis en las producciones de significados del lenguaje, a través de los cuales los seres humanos construyen su mundo (Banchs, 2000).

La influenza se ha estudiado desde el enfoque de las RS, se realizó una investigación en el área metropolitana de la ciudad de México, con una muestra de niños y adolescentes. Las creencias de la influenza tipo A, en su mayoría, fueron en torno a las formas de contagio y a las acciones preventivas. Se detectaron, además, teorías del complot; son teorías o rumores que aparecen cuando hay eventos que generan una fuerte tensión social; es información especulativa no confirmada, que condiciona el comportamiento de los individuos (Osorio, Osorio, \& Osorio, 2011).

Otro estudio realizado, también en la ciudad de México, tuvo por objetivo evaluar el impacto psicosocial de la enfermedad en profesionales de la salud, en personas con influenza y otras enfermedades respiratorias, así como a sus familiares. Las principales reacciones emocionales reportadas por los participantes fueron la incertidumbre y el miedo. Las personas que vivenciaron la enfermedad a través de algún ser querido o familiar mostraron un mayor grado de sensibilización sobre el contagio y las medidas preventivas (Romero, Gaál, Pick, Barriguete, Navarro, Porteny, \& Cervantes, 2009 ).

Un estudio más aporta la visión de futuros profesionales de la salud y de docentes de ciencias de la salud. Era una investigación de consenso cultural y de las dimensiones conceptuales de la influenza A (H1N1). Las dimensiones que favorecen la prevención del problema son los conceptos de influenza, síntomas, mecanismo de transmisión, cuidados sugeridos y su atención. Las dimensiones que pueden obstaculizar la prevención son la falta de credibilidad en las autoridades y en la información ambigua transmitida por los medios de comunicación (Torres-López, Matsui, \& Aranda, 2012).
Un estudio realizado en China sobre conocimientos, actitudes y prácticas sobre la influenza, según población de zona urbana y rural, reporta que el conocimiento a respecto de la enfermedad es confuso; mientras que, las actitudes y prácticas positivas para su prevención solo se reportan por mujeres con alto nivel educativo, lo cual está asociado a una alta percepción al riesgo de contagio (Lin, Huang, Nie, Liu, Yu, Yan, \& Xu, 2011).

Otro estudio, realizado en India, reporta que la población percibe a la influenza como una enfermedad grave y reconoce la eficacia de las medidas preventivas. Las mujeres mostraron un mayor conocimiento sobre la enfermedad y actitudes positivas hacia la prevención. Sin embargo, la mitad de los participantes no manifestó confianza en las autoridades gubernamentales ni en las medidas tomadas por estas durante la epidemia (Kamate, Agrawal, Chaudhary Singh, Mishra, \& Asawa, 2010).

Considerando estas ideas, en este estudio se pretende dar respuesta a la pregunta de investigación ¿cómo son las RS de la influenza humana por parte de adolescentes de la ciudad de Guadalajara, México, en dos épocas diferentes: durante la epidemia del 2009 y a inicios del año 2016, cuando se presentó un brote de influenza?

\section{Método}

El diseño de estudio comprendió dos fases: la estructura y el contenido de las RS de la influenza humana: a) Estructura a través de la identificación del nodo central empleando el criterio de saliencia, con base en el análisis prototípico (Verges, Tyszka, \& Verges, 1994); b) Contenido de la RS con base en el análisis procesual cualitativo, a través del análisis de categorías temáticas emergidas del discurso de los participantes (Banchs, 2000).

\section{Participantes}

El estudio se realizó en la ciudad de Guadalajara, México en una zona de clase media. Se integró una muestra de tipo propositiva (Suri, 2011). Los criterios para su selección fueron: adolescentes estudiantes de bachillerato, habitantes de la ciudad de Guadalajara e 
inclusión de ambos sexos. Fueron abordados fuera de una institución pública de educación media superior.

Los participantes fueron 68 mujeres y 44 hombres (112 personas en total), sus edades fluctuaron entre los 15 y los 18 años, con un promedio de 17 años en las mujeres y 16.8 años en el caso de los hombres. El 26.8\% reportó conocer a alguna persona que padeció influenza.

\section{Instrumentos}

La técnica de recolección de datos fue asociativa, a través de listados libres; estos favorecen la expresión espontánea de los sujetos y, por ello, se espera que el contenido evocado esté más libre de racionalizaciones, sesgos de defensa o deseabilidad social (Ruiz, Ponce de León, \& Herrera, 2001). Consisten en pedir a los informantes términos relacionados con una esfera conceptual particular: "Dime cinco palabras o frases que se te vienen la mente cuándo escuchas: influenza humana", una vez escritas las palabras en el instrumento, se les pide una pequeña explicación de por qué dijeron cada una de ellas. El tiempo de aplicación fue de cinco a diez minutos, lo que facilitó la participación de las personas.

\section{Estrategia de Analisis}

Se realizaron dos tipos de análisis:

a) Análisis prototípico: Se identificó la salience de cada término a través de la obtención del índice de Smith ${ }^{1}$ (1993). Este cálculo se basa tanto en la frecuencia de mención del término como en el rango de la ubicación que le otorgó cada informante a cada palabra (de tal forma que los primeros mencionados en el listado, tienen un mayor peso que los últimos). Para su obtención, se utilizó el software Anthropac v. 4.98. Se procedió a clasificarlos considerando la frecuencia y el rango promedio obtenido en los cuatro grupos de participantes (hombre y mujeres; datos del 2009 y del

\footnotetext{
${ }^{1}$ Por cada listado libre individual se calcula:

$\mathrm{s} j=1 \mathrm{r} j 1$

$\mathrm{n} 1 \quad \mathrm{n} 1$

Donde $r_{j}=$ posición del ítem $j$ en la lista, $\mathrm{y} \mathrm{n}=$ número de ítems de la lista. Para obtener el índice de salience, es necesario obtener el promedio de sj de todos los respondientes (Smith, 1993).
}

2016). Las palabras que constituyen el núcleo central de una RS serían aquellas que son citadas más veces y en primer lugar (rango) (Ruiz et al., 2001).

b) Análisis procesual: Categorización de las definiciones dadas para cada palabra, lo cual se hizo con análisis de contenido temático (Minayo, 1995) con el apoyo del software Atlas.ti.

\section{Confiabilidady validez de la información}

Se realizó triangulación por contrastación de técnicas y de investigadores (Flick, 2014), ya que en el proceso de análisis participaron todos los investigadores en forma separada, quienes llegaron a los mismos resultados.

\section{Consideraciones éticas}

Antes de cada entrevista, se explicaron los objetivos del estudio y se obtuvo el consentimiento informado verbal, garantizándoles a los informantes la confidencialidad y anonimato en la recolección y el análisis de la información. La participación de los entrevistados y entrevistadas fue voluntaria. El proyecto de investigación que fundamentó el presente estudio fue evaluado y, en su momento, aprobado por el comité de ética del Departamento de Salud Pública de la Universidad de Guadalajara, México (registro IISO/CI/03/10).

\section{Resultados \\ Análisis prototípico: Organización de las representaciones sociales de la influenza}

Se retuvieron las palabras que fueran evocadas por lo menos por dos personas. En el prototipo de la RS, los elementos hipotéticamente centrales son aquellos que son más frecuentes y evocados en los primeros lugares de la lista de palabras. En la tabla 1 se presenta el prototipo (frecuencia x rango de evocación) de la representación social de influenza de adolescentes en el año 2009.

En el caso de los hombres, el rango de evocación retenido fue del $56 \%$ y el rango promedio fue de 3.2. La palabra identificada como núcleo central es "enfermedad". Los términos ubicados como sistema periférico solo fueron dos: "ignorancia" y 
Tabla 1

Prototipo (frecuencia $\times$ rango de evocación) de las representaciones sociales de la influenza de adolescentes, 2009

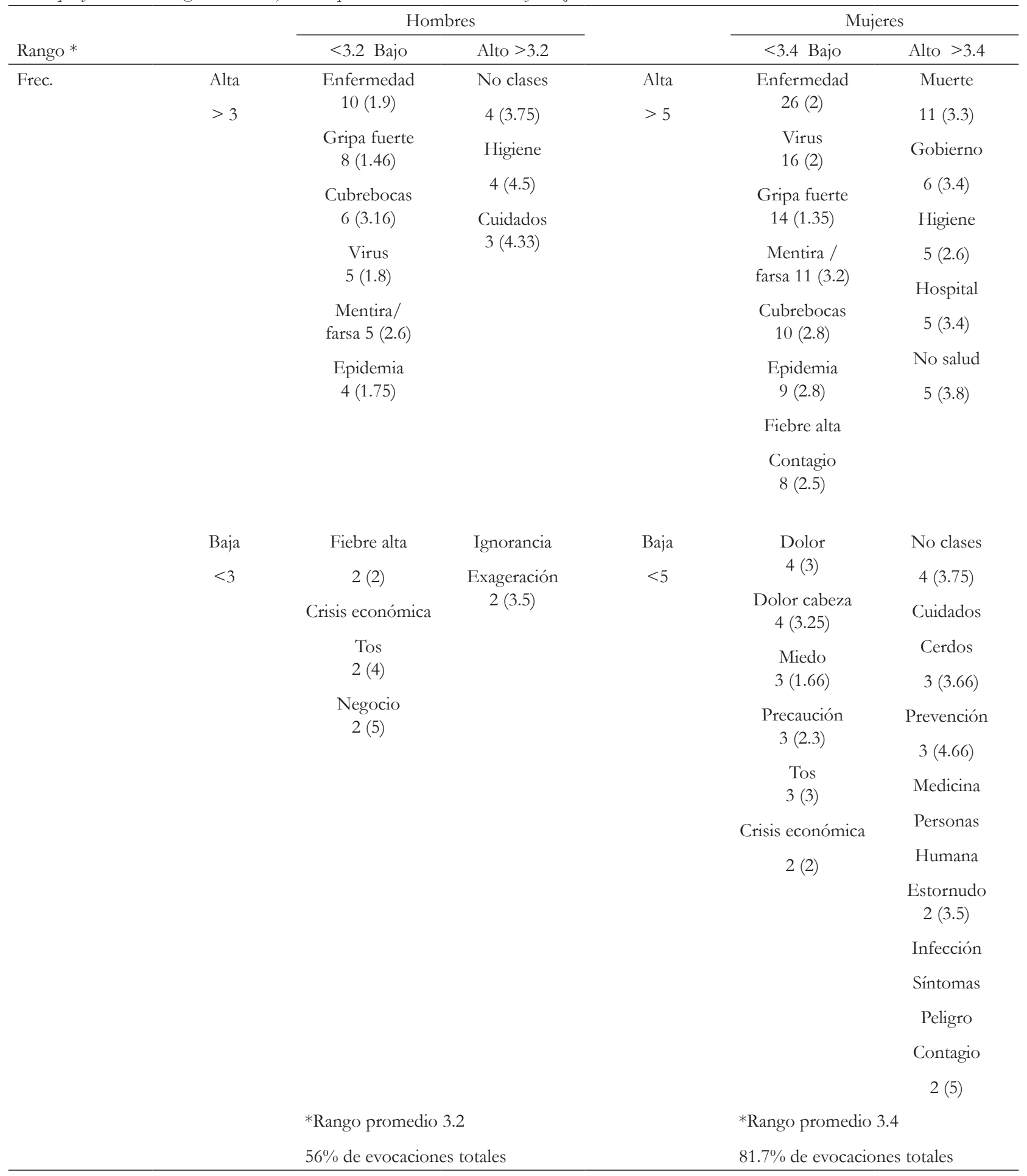


Tabla 2

Prototipo (frecuencia $\times$ rango de evocación) las representaciones sociales de la influenza de adolescentes, 2016

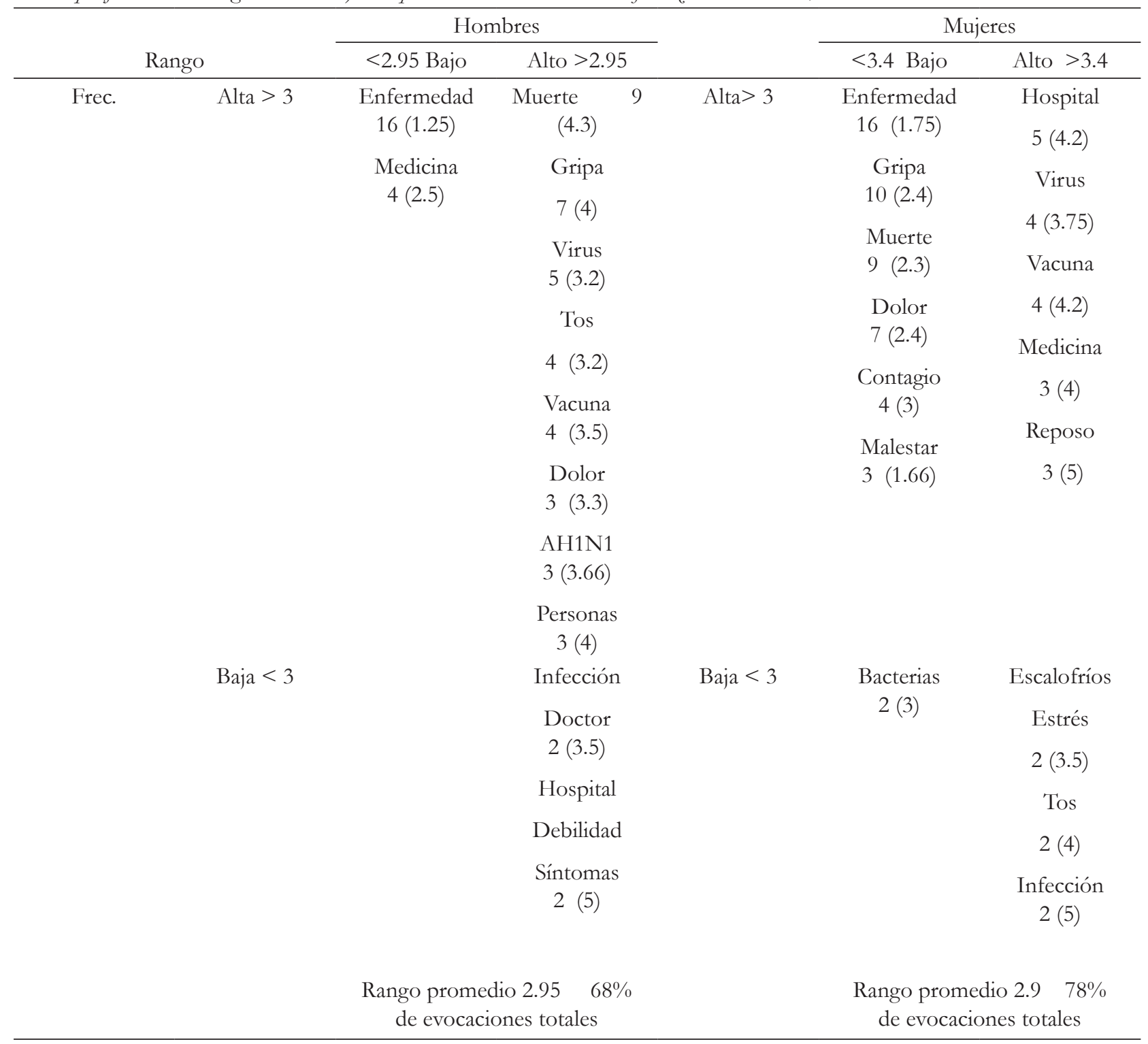

"exageración". En el caso de las mujeres, el rango de evocación retenido fue del $81.7 \%$ y el rango promedio fue de 3.4. Los términos ubicados en una frecuencia alta $(>5)$ y un rango bajo $(<3.4)$ son los que constituyen el núcleo central de la RS.

Para este caso, es la palabra "enfermedad", otros términos ubicados en este cuadrante (superior izquierdo) hacen referencia a otros componentes de la enfermedad. Los términos ubicados en el recuadro de frecuencia $(<5)$ baja y rango alto $(>3.4)$ (inferior derecho) son considerados el sistema periférico de la RS. Estos incluyen elementos asociados a la enfermedad, a los síntomas, a la prevención, a efectos secundarios de la contingencia, a un calificativo y a 
un ícono representativo en dicho momento como causante de la enfermedad (cerdos). En la tabla 2 se presenta el prototipo (frecuencia $x$ rango de evocación) de la representación social de influenza de los adolescentes en el año 2016.

Para el caso de los hombres, el rango de evocación retenido fue del $68 \%$ y el rango promedio fue de 2.95. La palabra identificada como núcleo central es "enfermedad", junto con el término "medicina". Los elementos periféricos se relacionan con "síntomas" y "debilidad"; un componente y dos más asociados a la atención de la enfermedad. Para las mujeres, el rango de evocación retenido fue del $78 \%$ y el rango promedio fue de 2.9. La palabra identificada como núcleo central es "enfermedad"; dentro de este grupo, se encuentran algunos síntomas, un componente "contagio" y una consecuencia "muerte". Los elementos periféricos son síntomas, un componente "infección" y una consecuencia de tipo emocional: "estrés".

Análisis procesual: El contenido de las representaciones sociales de influenza

Las categorías de análisis encontradas fueron: síntomas y consecuencias, transmisión de la enfermedad, tratamiento, prevención, reacciones emocionales, adjetivos otorgados y términos asociados.
Las categorías temáticas encontradas para la RS de influenza en el año 2009 se presentan en la tabla 3.

Categorías:

a) Transmisión: El término más evocado fue enfermedad, definida como una infección de tipo respiratorio, de actualidad, causó una epidemia y mucho alboroto, requiere de atención médica y hospitalaria. Es causada por un virus nuevo (o mutado), peligroso y contagioso. El contagio debe prevenirse, porque el virus se distribuye fácilmente por medio de la saliva. Es una epidemia porque se propaga con rapidez, y ya es pandemia porque "ya está en muchos países". Si no se detecta y trata oportunamente, puede provocar la muerte, ya ha causado muchas muertes.

b) Síntomas: Las manifestaciones de la influenza son: gripa fuerte, fiebre alta, dolor en general y dolor de cabeza en específico, tos, estornudo, cansancio o debilidad, bajas defensas, cuerpo cortado, flujo nasal, vómito, diarrea y malestar general.

c) Términos asociados: Se duda de la existencia de la enfermedad y no se cree en los datos estadísticos sobre morbilidad y mortalidad reportados por los medios oficiales. Los políticos se aprovechan de la situación, para implementar leyes y las personas no se pueden manifestar en contra. Existe la posibilidad de que sea

Tabla 3

Categorías encontradas en el análisis de la palabras obtenidas en los listado libres para la representaciones sociales de la influenza, 2009

\begin{tabular}{|c|c|c|c|c|c|c|}
\hline \multirow[t]{2}{*}{ Categoría } & \multicolumn{2}{|c|}{ Mujeres } & \multicolumn{2}{|c|}{ Hombres } & \multicolumn{2}{|c|}{ Total } \\
\hline & Frecuencia & $(\%)$ & Frecuencia & $(\%)$ & Frecuencia & $(\%)$ \\
\hline Transmisión & 79 & 23.6 & 24 & 7.0 & 103 & 30.5 \\
\hline $\begin{array}{l}\text { Síntomas y } \\
\text { consequencias }\end{array}$ & 51 & 15.2 & 19 & 5.4 & 70 & 20.7 \\
\hline $\begin{array}{l}\text { Términos } \\
\text { asociados }\end{array}$ & 41 & 12.2 & 25 & 7.2 & 66 & 19.5 \\
\hline Prevención & 32 & 9.6 & 23 & 6.4 & 55 & 16.4 \\
\hline Adjetivos & 9 & 2.6 & 11 & 3.1 & 20 & 5.9 \\
\hline $\begin{array}{l}\text { Reacciones } \\
\text { emocionales }\end{array}$ & 8 & 2.4 & 8 & 2.1 & 13 & 3.8 \\
\hline Tratamiento & 8 & 2.4 & 3 & 0.8 & 11 & 3.2 \\
\hline Total & 230 & $68.0 \%$ & 108 & $32.0 \%$ & 338 & $100 \%$ \\
\hline
\end{tabular}


un invento o estrategia política, hay crisis económica porque la influenza está causando que cierren los comercios, además de afectar al turismo.

d) Prevención: Es importante tomar medidas y se debe tener cuidado al tratar con la gente. Tenemos que ser muy cuidadosos para no contagiarnos usando las medidas preventivas (sea verdadera o no la presencia de la enfermedad). Las principales medidas son la higiene, el uso de cubrebocas y desinfectante, no dar besos, no automedicarse y respetar la cuarentena.

e) Adjetivos: es un invento, una broma, un fraude, apoyado en la ignorancia de las personas, provocando una atención exagerada al tema, caos y escándalo mundial. Hay confusión sobre si existe o no, es una enfermedad grave, es una enfermedad muy peligrosa y puede causar la muerte. Es un virus nuevo, se le identifica como humana "porque se contagia de humano a humano".

f) Reacciones emocionales: fueron calificadas de negativas así como de exageradas. Las negativas destacaron al miedo, pánico, angustia, estrés y preocupación. Las señalas como exageradas fueron histeria colectiva y paranoia.

g) Tratamiento: Es necesario tomar medicamentos para estar bien. Se considera que "la industria farmacéutica estaba en quiebra y con esto comenzaron a recuperarse sus ventas".

Las categorías temáticas encontradas para la RS de influenza en el año 2016 se presentan en la tabla 4.

Categorías:

a) Síntomas y consecuencias: ahogo, cansancio, debilidad, dolor, escalofrío, estornudos, fiebre, gripa, malestar, mareo, mocos, tos y asco. La mala salud se genera por no cuidarse. La muerte se presenta porque "hay muchas personas que no se cuidan y no son atendidas a tiempo y mueren".

b) Transmisión: Es una enfermedad; se puede confundir con otras; es dañina, difícil, severa, grave, peligrosa, contagiosa, dolorosa; afecta al sistema inmunológico; si no se cuida, puede ser más grave. Es una infección, un virus A (H1N1), una plaga. Es muy contagiosa, en las personas "es donde se almacena el virus" y ya se convirtió en una pandemia o epidemia. Se señalaron las bacterias que incluye y al mosquito como un trasmisor de la misma y el invierno como la época del año en la cual se presentan más casos de la enfermedad.

c) Tratamiento: se identificó al médico como la persona "que diagnostica si tienes dicha enfermedad", al hospital como un espacio de atención, a fin de evitar una emergencia y otras complicaciones. El tratamiento es la medicina a través de inyecciones. Recomendaron también reposo.

d) Prevención: La principal medida de prevención citada es la vacuna, otras incluidas fueron gel antibacterial y el cubrebocas.

e) Adjetivos: La influenza es un problema y un riesgo.

f) Reacciones emocionales: estrés, mal humor, molestia, preocupación, sufrimiento y tensión.

g) Otras palabras asociadas: influencia, cerdos, rock, sangre e inmune.

La triangulación de los hallazgos en ambos métodos se presenta en la tabla 5 .

Durante la epidemia del año 2009, las RS de la influenza parecen ir desde la identificación de la enfermedad con la incredulidad de su existencia.

Es una enfermedad de tipo respiratorio, parecida a una gripa fuerte, producida por un virus nuevo, contagioso que ha generado una epidemia y pandemia. Las formas de transmisión son claras y también los síntomas que genera en las personas. No obstante, se duda de la gravedad de la situación, calificándola de una farsa o mentira; consideran que fue usada por el gobierno como estrategia para ocultar sus movimientos. Generó reacciones emocionales negativas en la población (miedo, estrés, pánico, etc.), problemas económicos y sociales. Las mujeres destacan más los síntomas y la importancia de los cuidados para evitar el peligro de muerte en caso de contagio, mientras que los hombres resaltan la idea de que las medidas tomadas para su control en el país son una exageración. La categoría de prevención se ubica en un lugar importante dentro de estas RS. 
Tabla 4

Categorías encontradas en el análisis de la palabras obtenidas en los listado libres para la representaciones sociales de la influenża, 2016

\begin{tabular}{|c|c|c|c|c|c|c|}
\hline \multirow[b]{2}{*}{ Categoría } & \multicolumn{2}{|c|}{ Mujeres } & \multicolumn{2}{|c|}{ Hombres } & \multicolumn{2}{|c|}{ Total } \\
\hline & Frecuencia & $(\%)$ & Frecuencia & $(\%)$ & Frecuencia & $(\%)$ \\
\hline $\begin{array}{l}\text { Síntomas y } \\
\text { consequencias }\end{array}$ & 29 & 14.2 & 48 & 23.5 & 77 & 37.7 \\
\hline Transmisión & 23 & 11.3 & 44 & 21.6 & 67 & 32.9 \\
\hline Tratamiento & 12 & 5.8 & 12 & 5.8 & 24 & 11.7 \\
\hline Prevención & 3 & 1.5 & 8 & 3.9 & 11 & 5.4 \\
\hline Adjetivos & 0 & 0 & 10 & 4.9 & 10 & 4.9 \\
\hline $\begin{array}{l}\text { Reacciones } \\
\text { emocionales }\end{array}$ & 7 & 3.4 & 8 & 2.1 & 13 & 3.8 \\
\hline $\begin{array}{l}\text { Términos } \\
\text { asociados }\end{array}$ & 0 & 0.0 & 6 & 3.0 & 6 & 3.0 \\
\hline Total & 74 & $36.2 \%$ & 130 & $63.8 \%$ & 204 & $100 \%$ \\
\hline
\end{tabular}

Tabla 5

Triangulación de hallazgos de las representaciones sociales de la influenza

\begin{tabular}{|c|c|c|c|c|}
\hline & \multicolumn{2}{|c|}{ Durante la epidemia 2009} & \multicolumn{2}{|c|}{ A siete años de la epidemia 2016} \\
\hline Análisis Prototípico & $\begin{array}{l}\text { Núcleo centrala: } \\
\text { enfermedad, } \\
\text { síntomas y mentira. } \\
\text { Hombres: epidemia. } \\
\text { Mujeres: contagio. }\end{array}$ & $\begin{array}{l}\text { Elementos } \\
\text { Periféricos }{ }^{\mathrm{b}} \text { : } \\
\text { Hombres: exageración } \\
\text { e ignorancia. } \\
\text { Mujeres: atención } \\
\text { y peligro. }\end{array}$ & $\begin{array}{l}\text { Núcleo central: } \\
\text { enfermedad. } \\
\text { Hombres: medicina. } \\
\text { Mujeres: síntomas } \\
\text { y muerte. }\end{array}$ & $\begin{array}{l}\text { Elementos Periféricos: } \\
\text { Hombres: síntomas } \\
\text { y atención de la } \\
\text { enfermedad. } \\
\text { Mujeres: estrés } \\
\text { y síntomas. }\end{array}$ \\
\hline $\begin{array}{c}\text { Análisis de contenido: } \\
\text { Categorías }\end{array}$ & $\begin{array}{l}\text { Más relevantes } \\
\text { + Transmisión: } \\
\text { rápida y peligrosa. } \\
\text { + Síntomas y } \\
\text { términos asociados: } \\
\text { duda, mentira. } \\
\text { + Prevención: } \\
\text { cubrebocas, } \\
\text { desinfectante, } \\
\text { distancia social. }\end{array}$ & $\begin{array}{l}\text { Menos } \\
\text { - Emociones: miedo, } \\
\text { histeria colectiva. } \\
\text { - Adjetivos: } \\
\text { invento, fraude. } \\
\text { - Tratamiento: } \\
\text { medicamentos e } \\
\text { industria farmacéutica. }\end{array}$ & $\begin{array}{l}\text { Más relevantes } \\
\text { + Síntomas y } \\
\text { consecuencias. } \\
\text { + Transmisión: } \\
\text { confundible, grave. } \\
\text { + Tratamiento: } \\
\text { medicina y } \\
\text { atención médica. }\end{array}$ & $\begin{array}{l}\text { - Emociones: estrés } \\
\text { y preocupación. } \\
\text { - Adjetivos: } \\
\text { problema y riesgo. } \\
\text { - Prevención: vacuna, } \\
\text { gel antibacterial } \\
\text { y cubrebocas. }\end{array}$ \\
\hline
\end{tabular}

${ }^{a}$ Núcleo central: sistema que da significado a las representaciones sociales.

'Los elementos periféricos: se caracterizan por estar más determinados por la historia individual de las personas y por sus experiencias particulares. 
En el año 2016, a siete años de la epidemia, las RS de la influenza muestran una mayor caracterización de la enfermedad, sus síntomas y su atención. Las RS de la influenza de los adolescentes son: una enfermedad o infección dañina y grave, producida por un virus contagioso, A (H1N1), que puede generar epidemias. Las formas de transmisión y los síntomas son señalados; es como una gripa grave o más fuerte, que requiere tratamiento y atención médicos.

Se identifica al ser humano como portador del virus y al invierno como la época en que se presenta con mayor frecuencia. Las mujeres destacaron los síntomas; la importancia de la atención y cuidados a fin de evitar el peligro de morir; y que tiene diferentes consecuencias emocionales, principalmente el estrés. Mientras tanto, los hombres destacaron el tratamiento con medicina y atención hospitalaria. En este caso, la categoría de prevención ocupa un lugar menos relevante e incluye a la vacuna para evitar la enfermedad.

\section{Discusión}

Durante la epidemia 2009: Entre la enfermedad y la incredulidad

Los adolescentes en 2009 señalaron que la epidemia era una mentira, al igual que lo reportado por Osorio, et. al (2011) en población infantil y adolescente de la ciudad de México. La supuesta inexistencia de la influenza implica una baja percepción del riesgo de contagio y representa una posible barrera para una adecuada atención a la emergencia; el caso contrario incluiría la sensación de vulnerabilidad y de la gravedad de la enfermedad, esto último tendría un valor motivacional en el proceso de toma de decisiones (Oblitas, 2006).

El que las mujeres destacaran tanto la sintomatología como la importancia de los cuidados podría ser reflejo del rol tradicional del género femenino en la atención de los problemas de salud. Lo que ya se reportó en Corea con mujeres con alta escolaridad (Park, Cheong, Son, Kim, \& Ha, 2010) y con mujeres hindús que mostraron una actitud positiva hacia las medidas a tomar ante la influenza (Kamate et al., 2010).
A siete años de la epidemia 2016: la enfermedad, sus sintomas y su atención

Las RS de la influenza, en el caso de los adolescentes de 2016, parecen haberse resignificado en torno a los síntomas, su atención y sus posibles consecuencias. Los términos ubicados tanto en el núcleo central como en el sistema periférico son congruentes, aun cuando se presentan algunas diferencias por género. Esto puede tener relación con el hecho de que los participantes conocen a personas que han padecido influenza, experiencia que enriqueció su visión en torno a sus manifestaciones. Con esto se manifiesta la función de las RS de la vulgarización de las teorías científicas (Ibáñez, 1994), donde el uso de terminología biomédica (por ejemplo, A (H1N1)) ya forma parte del discurso de los adolescentes. Además, las RS de la influenza en este grupo de adolescentes contienen lenguaje biomédico, incluyen información, en su mayor parte correcta, sobre la sintomatología y transmisión de la enfermedad (excepto en el caso de una joven que señala la transmisión a través del piquete de un mosquito). Esto puede tener relación con el tipo de información que circula en los medios en torno a la enfermedad, ya que se ha reportado que el conocimiento de la influenza es derivado de un acceso a diversas fuentes de información, en las que se destacó a la televisión como el principal medio masivo difusor (Valdez, Díaz, \& Huerta, 2010; Torres-López et al., 2012).

Coincidencias entre las RS de los adolescentes de 2009 y de 2016

Las coincidencias entre las RS de la influenza de los adolescentes durante la epidemia y siete años después, son la identificación de la influenza como una "gripa fuerte" e inclusión de medidas preventivas.

La asociación de la influenza con la gripa constituye otra de las funciones de las RS: permitir integrar nuevos conocimientos dentro de un marco comprensible y coherente con los valores de los adolescentes (Ibáñez, 1994). El uso de esa analogía facilita la incorporación de una enfermedad, considerada como "nueva" en el año 2009, en su conocimiento de sentido común de 
los procesos de salud y enfermedad. Así, la cultura influirá sobre los significados que adscriben las personas a los síntomas, al igual que a la experiencia previa, las creencias y las representaciones de las enfermedades en general (Morrison \& Bennett, 2008).

Se incluyeron pocos términos relacionados con la prevención del padecimiento y los adolescentes de 2016 la ubican en un lugar poco importante en la estructura de las RS de la influenza. A las medidas citadas en común (gel, cubrebocas, uso de otro tipo de barreras), se agregó la vacunación en el caso de los adolescentes de 2016, ya que la de 2009 no incluía la cepa A (H1N1).

Una más de las funciones de las RS, es que guían los comportamientos y las prácticas sociales; prescriben cómo actuar y en qué ocasiones (Ruiz et al., 2001). Por lo tanto, al no destacar las medidas preventivas, es de suponer que lo adolescentes difícilmente las llevarán a cabo. Por otro lado, los sesgos optimistas conducen a subestimar los riesgos objetivos y a no poner en práctica ciertas conductas precautorias para evitar consecuencias nocivas para la salud (Oblitas, 2006). Las posibles barreras para la prevención de la influenza que deben combatirse con información científica suficiente y adecuada.

Diferencias entre las $\mathrm{RS}$ de la influenza de los adolescentes de 2009 y de 2016

Las diferencias encontradas en las RS de la influenza en los dos períodos de tiempo estudiados son el tipo de reacciones emocionales ante la enfermedad, el tratamiento y los adjetivos otorgados al padecimiento.

En las reacciones emocionales durante la epidemia se señala al miedo, lo que podría ser un arma de dos filos en la prevención de la influenza. Esto porque, por un lado, un nivel de ansiedad puede promover cambios positivos en las conductas de prevención (Goodwin, Haque, Neto, \& Myers, 2009). Por otro lado, un nivel elevado puede conducir a la paralización de la población ante una amenaza considerada inevitable (Barrera, 2010) y, por tanto, la percepción de autoeficacia de las acciones preventivas de los adolescentes podría ser baja o inexistente.
En el caso de los adolescentes en 2016, las reacciones emocionales van más hacia las incomodidades que ocasionan los cuidados que implica la enfermedad (mal humor, molestia, tensión), pero el miedo ante el posible contagio no fue incluido. El miedo ha sido usado por los promotores de la salud y los políticos que financian sus actividades como una forma de aumentar la influencia de la comunicación mediática, tal vez porque se ajusta a las creencias implícitas acerca de su influencia sobre la conducta. Sin embargo, se ha demostrado que es relativamente ineficaz para provocar un cambio de conducta (Oblitas, 2006).

La segunda diferencia es sobre el tipo de tratamiento. Durante la epidemia, el uso de medicamentos obtuvo valoraciones bajas, además de críticas hacia el papel de la industria farmacéutica. Mientras tanto, en 2016, ocupa un lugar más importante y se centra en el aspecto médico (atención hospitalaria y medicina).

La tercera diferencia es en los calificativos atribuidos a la enfermedad; durante la epidemia, se centraron en la incredulidad de su existencia a la vez que, en forma contradictoria, se considera grave y peligrosa. Como ya se mencionó, la percepción de un bajo riesgo al contagio, como sucedió durante la epidemia, puede llevar a relajar las medidas de prevención (Apiñaniz, López-Picado, Miranda-Serrano, Latorre, Cobos, et al., 2010 ; Cowling, Ng, Ip, Liao, Lam, Wu, et al., 2010). En el año 2016, los adjetivos también fueron negativos, pero denotando ser un peligro y un riesgo para la salud; es decir, ya se le visualiza como un problema de salud existente.

\section{Propuestas desde la Psicología de la Salud}

Es común que los temas sobre la psicología de salud de los adolescentes se centre en sus prácticas sexuales, los accidentes y el consumo de substancias (Phillips, 2003), mas los temas que abordan sus conocimientos y creencias sobre enfermedades emergentes, como es el caso de la influenza, son poco considerados. Las representaciones sobre la enfermedad son concepciones organizadas de enfermedades concretas, adquiridas a través de los medios y la experiencia personal. Independientemente de cómo 
sean, influyen sobre la conducta preventiva y sobre las respuestas de afrontamiento de los individuos que experimentan síntomas o enfermedades (Morrison \& Bennett, 2008).

La primer propuesta que se desprende del estudio realizado es referente al diseño de campañas de salud informativas, tomando como base el modelo de creencias sobre la salud (Rosenstock, 1974; Becker, 1974; Strecher, Champion; Rosenstock, 1997 en Morrison \& Bennett, 2008). Este modelo propone que la probabilidad de que una persona realice determinada conducta de salud depende de factores demográficos (sexo, edad, clase social, etc.) y de una serie de creencias que pueden surgir de determinada clave para la acción, interna o externa. Estas creencias incluyen las percepciones de la amenaza y la evaluación de la conducta en cuestión; añadiéndose, posteriormente, las claves para la acción y la motivación para la salud (Morrison \& Bennett, 2008).

A la vez, se deben considerar los subprocesos cognitivos implicados en cualquier campaña dirigida promover el cambio en las actitudes y creencias de los adolescentes con respecto a su salud. Esto incluye la captación de la atención de las personas a las cuales va dirigida: a) Comprensión del mensaje, b) Aceptación de propuestas, c) Retención del mensaje y d) Acción efectiva: cambio hacia conductas saludables (Oblitas, 2006).

La segunda propuesta se orienta a promover la autoeficacia y autocontrol de los adolescentes en sus cuidados de salud. La primera puede entenderse como la creencia de que uno puede presentar determinada conducta en determinadas circunstancias (Morrison \& Bennett, 2008). La autoeficacia percibida determina la cantidad de esfuerzo invertido y el nivel de perseverancia. Las personas que confían en sí mismas, que se sienten capaces de llevar a cabo los cambios propuestos para mejorar su salud, persisten en mantener el curso de sus acciones; a pesar de los obstáculos, perciben alternativas de solución estos y se recuperan más fácilmente de las frustraciones (Oblitas, 2006).
Por su parte, el autocontrol se refiere a la capacidad de las personas para regular su conducta. Gran parte de los comportamientos del ser humano está motivada y regulada por criterios internos y reacciones autoevaluadoras ante sus propias acciones y que todo acto incluye en sus determinantes las influencias autoproducidas. Las personas se proponen normas, objetivos, niveles de ejecución, que funcionan como motivadores de la conducta actual (Oblitas, 2006).

La tercer propuesta es la incorporación de la educación entre pares. Consiste en que se entrena a alumnos influyentes de un contexto (usualmente el escolar) sobre determinada cuestión sanitaria y se les anima a educar a sus compañeros sobre estas cuestiones, con la intención de que se promueva una conducta saludable. Los métodos utilizados pueden ser impartir un tema en forma de conferencia, en tutorías informales en entornos sin estructurar o en conversaciones y asesoramiento de uno a uno. Se considera que las ventajas de los programas de educación entre pares derivan del hecho de que los compañeros constituyen una fuente de información más creíble que los programas de educación habituales. La educación por los compañeros también ofrece la oportunidad de delegar competencias a los implicados y de que estos actúen como modelos positivos (Morrison \& Bennett, 2008).

\section{Limitaciones y fortalezas}

Las limitaciones del presente estudio se encuentran en la muestra seleccionada, ya que solo se estudió el punto de vista de la población universitaria, sin llegar a considerar la población adolescente que abandonó los estudios; esto porque en China se encontró que a mayor escolaridad la población tiene más cuidados (Lin, et al., 2011). Por ello, se sugiere, en futuros estudios, el empleo de muestreos estratificados. La segunda limitación es que, si bien se accedieron a las RS de los jóvenes, no se exploraron las prácticas de prevención y protección que realmente llevaron a cabo ante la influenza.

Las fortalezas del estudio son que se accedió al punto de vista de los adolescentes, población creciente 
en México y que fue reconocida como un grupo etario vulnerable durante la epidemia (ya que afectó más a jóvenes). Además, las aportaciones de la Psicología de la Salud para la implementación de programas preventivos y de promoción de la salud, considerando los procesos psicológicos de las poblaciones a las cuales se dirigen.

\section{Referencias}

Abric, J. C. (2001). Metodología de Recolección de las Representaciones Sociales. En Prácticas y Representaciones Sociales. México: Ediciones Coyoacán.

Apiñaniz, A., López-Picado, A., Miranda-Serrano, E., Miranda-Serrano, E., Latorre, A., Cobos, R. et al. (2010). Estudio transversal basado en la población sobre la aceptabilidad de la vacuna y la percepción de la gravedad de la gripe A/H1N1: opinión de la población general y de los profesionales sanitarios. Gaceta Sanitaria, 24 (4), 314-320.

Banchs, A. M. (2000). Aproximaciones procesuales y estructurales al estudio de las representaciones sociales. Papers on social representations, 9, 3.1-3.15.

Barrera, J. A. (2010). El miedo colectivo: el paso de la experiencia individual a la experiencia colectiva. El Cotidiano, 25(159): 5-10. Recuperado de: http:// www.redalyc.org/pdf/325/32512747002.pdf

Cowling, B. J., Ng, D. M., Ip, D. K., Liao, Q., Lam, W. W., Wu, J. T., et al. (2010). Community psychological and behavioral responses through the first wave of the 2009 Influenza A(H1N1) pandemic in Hong Kong. The Journal of Infectious Diseases, 202(6), 867-876.

Flick, U. (2014). La gestión de la calidad en investigación cualitativa. Madrid: Ediciones Morata.

Goodwin, R., Haque, Sh., Neto, F., \& Myers, L. B. (2009). Initial psychological responses to Influenza A, H1N1 ("Swine flu"), BMC Infectious Diseases, 9(166). Recuperado de: http:// bmcinfectdis. biomedcentral.com/articles/10.../1471-2334-9-166

Ibáñez, T. (1994). Psicología Social Construccionista. México: Universidad de Guadalajara.
Jodelet, D. (1993). La representación social: fenómenos, concepto y teoría. En Moscovici Serge. Psicología Social II. Barcelona: Paidós.

Kamate, S. K., Agrawal, A., Chaudhary, H., Singh, K., Mishra, P., \& Asawa, K. (2010). Public knowledge, attitude and behavioural changes in an Indian population during the Influenza A (H1N1) outbreak. Journal of Infection in Developing Countries, 4 (1), 007-014.

Lin, Y., Huang, L., Nie, S., Liu, Z., Yu, H., Yan, W., \& Xu, Y. (2011). Knowledge, Attitudes and Practices (KAP) related to the Pandemic (H1N1) 2009 among Chinese General Population: a Telephone Survey. BMC Infectious Diseases, 11, 128. doi: 10.1186/14712334-11-128

Minayo, C. (1995). El desafío del conocimiento. Investigación cualitativa en salud. Buenos Aires: Lugar Editorial.

Morrison, V. \& Bennett, P. (2008). Psicología de la Salud. Madrid, España: Pearson Educación, S.A.

Oblitas, L. A. (2006). Psicología de la salud y calidad de Vida. Ciudad de México, México: Thomson.

Organización Mundial de la Salud (OMS). (2009). Influenza A(H1N1) update 31. Global Alert and Response (GAR). Recuperado de: http://www.who. int/csr/don/2009_05_17/en/index.html

Organización Mundial de la Salud (OMS). (2014). Influenza virus infections in bumans (February 2014). Recuperado de: http://www.who.int/influenza/ human_animal_interface/virology_laboratories_ and_vaccines/influenza_virus_infections_ humans_feb14.pdf?ua $=1$

Organización Panamericana de la Salud (OPS). (2011). Preparación y respuesta ante la eventual introducción del virus chikungunya en las Américas. Washington D.C, EUA: OPS.

Organización Panamericana de la Salud - Organización Mundial de laSalud(OPS-OMS). (2016). Actualización epidemiológica. Actividad de influenza en la temporada 2015-2016. Recuperado de: http://www.paho.org/ hq/index.php?option $=$ com_docman\&task $=$ doc_vi ew\&Itemid $=270 \&$ gid $=33169 \&$ lang $=$ es. 
Osorio, M.; Osorio, M. A.; Osorio, M. G. (2011). Representación infantil de la influenza A, a través de un dibujo a tema. Psicolatina, 22, 1-13.

Park, J. H., Cheong, H. K., Son, D. Y., Kim, S., \& Ha, Ch. (2010). Perceptions and behaviors related to hand hygiene for the prevention of H1N1 influenza transmission among Korean university students during the peak pandemic period. BMC Infectious Diseases, 10, 222. doi: 10.1186/1471-2334-10-222

Phillips, Sh. (2003). Adolescent Health. En A.M. Nezu, Ch.M. Nezu, P.A. Geller (eds.). Health Psychology. Vol. 9. (pp. 465-485). New Jersey, USA: John Wiley $\&$ Sons.

Romero, A., Gaál, F., Pick, S., Barriguete, A., Navarro, C., Porteny, T., \& Cervantes, A. (2009). Impacto Psicosocial de la Epidemia de Influenza A(H1N1) en Pacientes, Familiares de Pacientes y Personal de Salud del Distrito Federal: Un Estudio Exploratorio. Reporte presentado al Centro Nacional de Prevención de Accidentes (CENAPRA). Recuperado de: http:// www.uaeh.edu.mx/investigacion/producto. php?producto $=2274$

Ruiz, J. I., Ponce De León, E., \& Herrera, A. N. (2001). Avances en Medición Evaluación en Psicología y Educación. Bogotá, Colombia: Universidad del Bosque.

Secretaría de Salud México. (2011). Sistema Nacional de Vigilancia Epidemiológica. Epidemiología. Sistema Único de Información. Informes epidemiológicos semanales 2011, 52(27). Semana 52, del 26 de diciembre al 1 de enero de 2011. Recuperado de: http:// www.epidemiologia.salud.gob.mx/informes/ informesh/2011/influenza/influenza11_52.html

Secretaría de Salud México (2016). Sistema Nacional de Vigilancia Epidemiológica. Epidemiología. Sistema Único de Información. Informes epidemiológicos semanales 2016, semana 20. Recuperado de: http:// www.epidemiologia.salud.gob.mx/informes/2016/ influenza-semanas.html

Smith, J. (1993). Using Anthropac 3.5 and a spreadsheet to compute a free-list salience index. Cultural Anthropology Methods Newsletter, 5(3), 1-3.

Suri, H. (2011). Purposeful sampling in qualitative research synthesis. Qualitative Research Journal, 11(2), 63 - 75.

Torres-López, T. M., Matsui, J. O. \& Aranda, C. (2012). Dimensiones culturales del concepto de influenza humana en estudiantes y docentes de ciencias de la salud que favorecen o dificultan su prevención. Desacatos, 39, 45-56.

Valdez, A., Díaz, S., \& Huerta, D. (2010). Influenza y Opinión Pública en Jalisco: Un Estudio Sobre las Percepciones Ciudadanas en Guadalajara, México. Pangea Revista de la Red Académica Iberoamericana de Comunicación, 1 (5), 98-107. Recuperado de: https://revistaraic.files. wordpress.com/2010/12/01-01-105.pdf

Verges, P., Tyszka, T., \& Verges, P. (1994). Noyau central, saillance et propriétés structurales. Papers on Social Representations, 3(1), 3-12. 